\title{
Study on 3D-IDS Based Spacecraft Development Mode
}

\author{
ZHOU Xiaolun*, CHEN Haifeng, HAO Ganggang, LUO Cheng, ZHU Wei
}

Beijing Institute of Spacecraft System Engineering, Beijing 100094, China

\begin{abstract}
The functions, applications, developments and current application mode of IDS $2 . x$ system are generally introduced in this paper. Then the development mode of spacecraft based on IDS2.x system is described. The existing problems especially the information redundancy of mechanical interface and their effects are pointed out. A new solution is proposed by developing 3D-IDS system. The central functions of 3D-IDS system are shown in this study. A new application mode of 3D-IDS system is explored and described by showing how to fill in, countersign and apply with 3D-IDS file. The 2D drawing and sketch are removed from 3D-IDS system to avoid information redundancy of mechanical interface. The consistency between 3D model and the parameters of IDS file can be guaranteed by the interface tool. The efficiency of filling in, countersigning and applying has been improved significantly, which greatly promotes the coordination and total efficiency of spacecraft system design departments and equipment design departments.
\end{abstract}

\section{Introduction}

IDS is the abbreviation of Interface Data Sheet in this paper. IDS file is a kind of important file which is used to control the mechanical, electrical and thermal interfaces between system, subsystem and equipment in spacecraft development. IDS files are the basis of spacecraft development engineering [1, 2, 3]. Initially, IDS files are managed manually. As the number of spacecraft development tasks increased continuously, it was becoming more and more difficult to meet the needs for manual management of IDS files. Therefore, IDS system was developed by China Academy of Space Technology around 2007. After more than ten years of application, IDS system has been developed V1.x version and V2.x version. The latest version is V2.5 version $[4,5]$.

The existing functions of IDS system meet the needs of spacecraft development to some extent, and realize the unified management of top-level data source. However, some problems cannot be fundamentally solved on IDS v2.x version. These problems focus on the mechanical interface. In IDS files, the mechanical interface includes parameter information, sketch, 2D drawing and 3D model. Parameter information is intuitive and readable, which is mainly used for information summary. Sketch

\footnotetext{
* Corresponding author: zhouxiaolun@cast.cn
}

is mainly used for non-mechanical professional designers to view the appearance of equipment. 2D drawings are mainly used to summarize the appearance atlas as the basis of equipment acceptance. 3D model is mainly used for system layout design. Parameter information, sketch, 2D drawing and 3D model exist simultaneously in IDS files, and mutual consistency is ensured by manual check. The review process is complex, inefficient and prone to inadequate verification. In addition, the 3D model provided by the equipment developer is usually difficult to be used directly for spacecraft layout design, which often requires a lot of re-processing and leads to repeated design.

IDS files can only be filled manually through IDS client. It does not support the identification and reading of model parameters, nor does it support the consistency comparison between IDS data and the model, which is prone to cause data inconsistency. To solve the problem, a 3D-IDS system and a new mode of spacecraft development based on 3D-IDS system is proposed in this paper.

\section{Current application modes for IDS system}




\subsection{IDS file overview}

IDS files usually consist of 13 types of sheets. These sheets include mechanical characteristics, sketch of equipment, thermal characteristics, thermal distribution, circuit, power supply, telemetry parameters, instructions, connectors, assignment of connectors, electrical characteristics, radio frequency interface and equipment description.

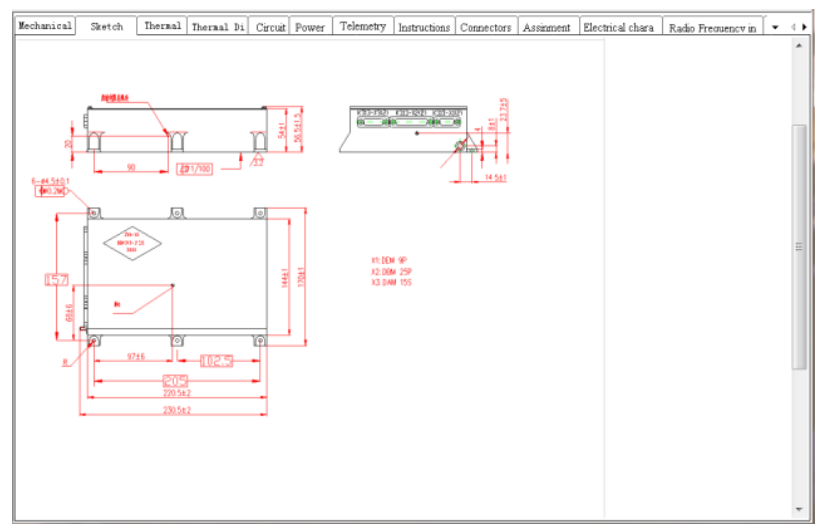

Fig. 1. Sheets of IDS file

Mechanical characteristics sheet includes outline size, mounting holes location, mounting lug size, mounting surface roughness, equipment weight, grounding specifications, and grounding number. The equipment sketch sheet includes the equipment outline diagram, the corresponding 2D drawing and 3D model.

The thermal characteristics sheet includes long-term heat consumption, instantaneous heat consumption, working temperature range, storage temperature range, surface absorptivity, surface emissivity and other parameters. The heat distribution sheet includes the heat consumption distribution diagram in the time domain and the heat consumption distribution diagram on the mounting surface.

The circuit sheet includes the principle of circuit, which reflects the principle of working circuit inside the equipment. The power sheet includes the power supply requirements of the equipment, including voltage range, ripple voltage characteristics, voltage stability, current characteristics and power consumption, etc.

Telemetry parameters sheet includes equipment telemetry parameters, such as the name of parameters, type, output level, output impedance and so on. The instructions sheet includes the instructions name, type, width, pulse amplitude, load impedance and other contents.

The electrical connector sheet includes the electrical connectors' code, name, type, pinhole and other contents. The distribution sheet of electrical connector node includes the type, direction, voltage, current, shielding requirements and other contents of each contact pin of every electrical connector.

The electrical interface characteristic sheet includes the signals characteristic and interface circuit of each interface signal. Radio frequency interface sheet includes high frequency signal connectors' type, code, direction, signal content, heat consumption and other content. The equipment description sheet includes the contents that especially need to be explained, such as special installation requirements, special accessories, special use requirements, etc.

IDS file is an organic whole, which comprehensively stipulates the mechanical, electrical and thermal interface requirements, input requirements, output requirements and use requirements of the equipment. Controlled IDS files are the basis for all kinds of design work, such as configuration design, layout design, pipeline design, cable network design, grounding design, information flow design, power supply design, assembly design, electrical design, thermal control design, etc.

\subsection{IDS file filling in and countersigning}

Each spacecraft is managed as a project, and all equipment of the project have their own IDS files, and all the IDS files of the same project are managed in the same project. Projects are managed by project managers. Project managers are responsible for daily management of the projects, including setting up the team, configuring rights, creating equipment, etc., to ensure that each sheet of IDS files has its own responsible person and countersign person, etc.

The lifecycle of IDS files can be roughly divided into three stages: environment preparation, filling in and countersigning; environment preparation mainly includes: creating a new project in IDS system, configuring IDS file template, setting team, setting personnel rights, setting countersigning mode, etc. According to equipment list, project manager will import the equipment information into IDS system. The information includes project code, stage, equipment code, equipment name and other contents. According to subsystem and research institute, the above information will be generated into the header information update package and sent to the corresponding research institute, which will create the IDS blank sheets according to the update package. 


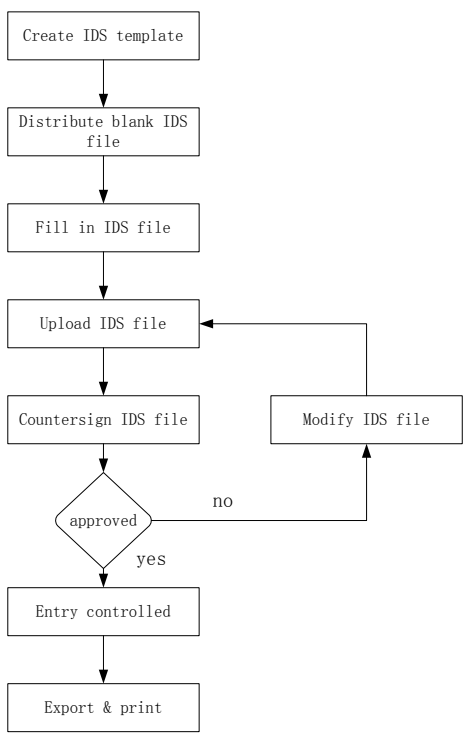

Fig. 2. Process of IDS system application

Based on the IDS client, the research institute designer will fill the blank sheet with detailed interface contents of equipment in accordance with "the unified requirements for filling in IDS file". Currently, the information of IDS files is mainly filled in manually. For example, the mechanical characteristics of the mechanical interface are manually filled in by the designer. 2D drawings and 3D models of the equipment are uploaded manually. When dealing with a large number of these IDS files, it is very easy to make mistake.

After each sheet of the IDS file has been filled in, the equipment supervisor will upload the IDS file to the IDS system and start the countersigning. The countersigner of each sheet will confirm the corresponding contents of the file, identify the contents that do not meet the requirements, and mark them in the file. The equipment designer will modify the content as indicated in the IDS file until all content meets the requirements of the countersigners. For the sheets that meet the requirements, the countersignature personnel will approve and sign electronically. After all sheets are passed, IDS files enter the controlled state.

\subsection{IDS file application}

IDS files are inputs for equipment, subsystems, and system development. The equipment development institute shall carry out the detailed design of the equipment based on the specific requirements in IDS. On the premise of meeting the relevant standards, it shall ensure that the mechanical, electrical and thermal interfaces conform to the provisions of IDS file.
The mechanical interface mainly focus on ensuring that the outline size, mounting holes location and weight are strictly consistent with IDS files. The electrical interface mainly focus on ensuring that the connector type, voltage, current and signal content of each pin are strictly consistent with IDS files. Thermal interface mainly focus on ensuring that the working temperature range, thermal distribution, and thermal consumption of the equipment are strictly consistent with IDS files.

Spacecraft designers carry out design work based on controlled IDS files, such as configuration design, layout design, cable network connection relationship design, cable network layout design, information flow design, power supply diagram design, grounding design, etc., all of which take IDS files as the design source.

The 3D models attached to the controlled IDS files are the main input for spacecraft layout design. Generally, the 3D models are detailed models, which take up a lot of storage space and cannot be directly used for the layout design. Spacecraft designers have to reduce the storage space of the equipment models in two ways: 1) using the system modeling tool to re-model; 2) deleting the information irrelevant in the equipment detailed model, and shrinking the envelope into an entity model. The advantage of the first method is that the storage space of the models decreases obviously and the models are convenient to use. The disadvantages of this method are: a) it is mainly applicable to more standard equipment, and is not suitable for irregular equipment; b) two sheets need to be added to the IDS file to describe the installation lugs and connectors of the equipment; the filling process and countersigning are complicated and inconvenient.

The advantages of the second method are: the processing is relatively simple, do not add IDS sheets, IDS filling in and countersigning process remains the same. The disadvantage is that when equipment model is processed in this way, the model storage space does not decrease significantly, which makes spacecraft model huge.

The design inputs of cable network connection relationship are mainly sheet 9 "electrical connector" and sheet 10 "electrical connector node assignment". The sheet 9 describes the connector list, code, type, usage, and corresponding electrical connector information of the equipment. The sheet 10 describes the number of pins for each electrical connector and the usage of each pin. The information provided by the two sheets supports the spacecraft designer to complete the design of the cable network.

The spacecraft designer imports the relevant information of IDS system into the cable network design system through the information interface between the cable network design system and IDS system, and then uses the design function of the cable network design system 
to complete the connection design of the low-frequency cable network. Other work such as equipment appearance atlas preparation, wood mold processing, information flow design, power supply and distribution diagram design, grounding diagram design, etc. all use IDS files as information sources.

\subsection{Disadvantages in the current mode}

There are some disadvantages in the current application mode of IDS system, which are increasingly unable to meet the requirements of automation, consistency and reliability. These disadvantages are mainly shown as follows:

a) Redundant information content and poor consistency. For example, mechanical interface information is expressed in four ways to meet the application needs of different scenarios: A) dimension parameters; B) sketch; C) 2D drawings; D) 3D models. Those kinds of information expression bring great difficulties to the filling in and countersigning of IDS files: relevant personnel need to constantly check the consistency of these types of information. The efficiency of filling in and countersigning is very low and prone to errors.

b) Low degree of automation. Manual verification of filling process and manual verification of countersigning process, lack of automatic tools as support. For example, the sketch sheet, the electric connector sheet and the electric connector sheet all contain the types of connectors respectively. Due to various reasons, IDS system cannot automatically identify and check the consistency of the information content of each sheet.

c) Much post-processing work. The 3D model and 2D drawing attached to the mechanical interface are detailed design uploaded by the equipment designer, which take up a lot of storage space and cannot be used for the spacecraft layout design directly. The model requires a lot of reprocessing work before using.

d) Invisibility of 3D models. The 3D model needs to be downloaded locally and can only be viewed through specified 3D software. Non-mechanical designers lack $3 \mathrm{D}$ software to view the equipment's $3 \mathrm{D}$ model, which is not convenient to use.

\section{Overview of 3D-IDS system functions}

With the continuous improvement of software and hardware, model-based digital design has increasingly become the development direction of industrial applications. After more than 20 years of unremitting development, the model-based definition (MBD) in the aerospace field has entered a new stage of comprehensive application and promotion. It has become an important way to improve the efficiency of design, production and test. How to realize the 3D information of IDS files is a technical problem that needs to be solved urgently. The demand of the 3D mechanical interface is most urgent, and the application effect will be the most significant. Beijing Institute of Spacecraft System Engineering takes the lead in proposing the concept, construction goals and technical approaches of 3D IDS. It is expected to preliminarily achieve the following goals through the construction of 3D IDS system:

a) Unique expression of mechanical interface information to avoid information redundancy;

b) Manual filling of mechanical interface is substituted by automatic filling based on 3D tools and models;

c) Automatic verification of mechanical interface information with 3D model;

d) $3 \mathrm{D}$ visualization of mechanical interface information in IDS files;

e) 3D model of IDS file is directly available in spacecraft system design.

The 3D IDS system is mainly realized by 3D design tool, IDS interface tool and IDS system. 3D design tool is used to open models, simplify models and set parameters. 3D-IDS interface tool is used to read parameter information of 3D model and upload 3D model into IDS file. The 3D- IDS file receives the parameter values from the model, such as weight, outline size, holes position, holes diameter, electrical connector position, grounding type, thermistor position and other information. It also provides real-time browsing of 3D models in IDS files.

Equipment sketch and 2D drawing attachments are removed in 3D-IDS files. The correlation between the $3 \mathrm{D}$ model and the mechanical characteristic parameter is realized so as to ensure that the values displayed in the IDS file sheets are consistent and correlated with the dimensions of the 3D model. It is very easy to fill in, countersign and apply the equipment interface information based on 3D model. IDS files can be verified by interface tool, which greatly reduces the work of countersignature. The model of the controlled IDS file can be downloaded and used directly, which greatly reduces the workload of model reprocessing. 


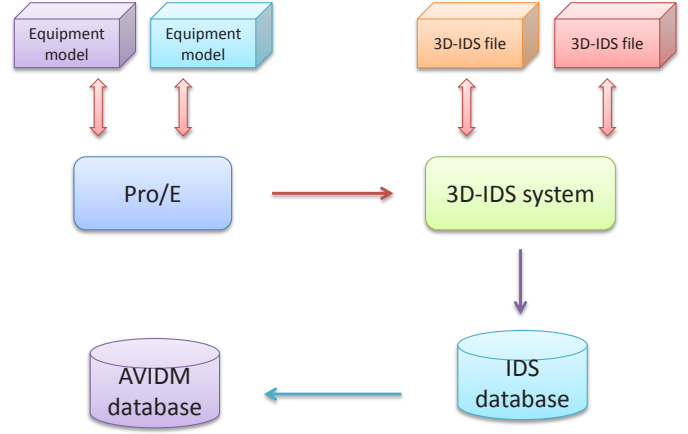

Fig. 3. Information flow of 3D-IDS

\section{Research and development mode exploration based on 3D-IDS system}

\subsection{IDS file filling in}

The filling way of mechanical interface will be changed greatly. From manual filling in parameter values, pasting equipment sketch, submitting 3D models, submitting 2D drawings and manual comparison, it has been transformed into standardized setting of model-based parameter, information reading and submitting based on interface tool.

3D model design of spacecraft equipment should be carried out according to the $3 \mathrm{D}$ model requirements of spacecraft equipment. The model should show the equipment body, lugs, mounting holes, connectors, grounding piles, high-low frequency cables, etc. which affect the appearance of the equipment. The model should be set corresponding parameter values such as weight, mass properties, etc.

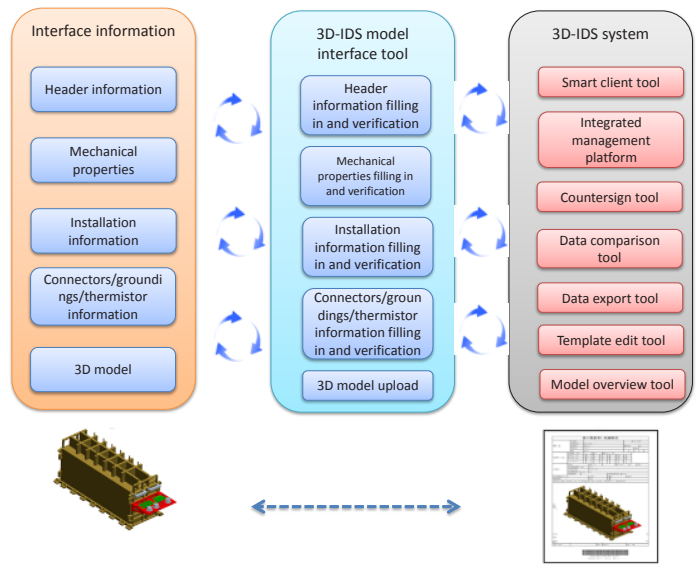

Fig. 4. Information flow of mechanical interface

After the model design is completed, the mechanical interface information in the 3D model is read and verified by interface tool. After the model information is verified, the interface information will be synchronized into IDS file. After the information is synchronized, the mechanical interface information in the model will be filled in the corresponding location of the mechanical interface sheet, and the 3D model are attached to the IDS file for display and transmission. If the mechanical interface information of the 3D model is not set completely, the model interface tool will not pass the verification, and the information cannot be filled in.

\subsection{D IDS countersigning}

After equipment designer finish filling in the mechanical interface, electrical interface, thermal interface and other relevant interface, the 3D-IDS file will be uploaded to IDS system uniformly by the subsystem designer. Then, 3D-IDS file will be reviewed by relevant system designers. For 3D-IDS files which are countersigned for the first time, the spacecraft designers only need to check whether the model storage size, installation interface and other contents meet the requirements, and do not need to check repeatedly whether the sketch, 3D model, 2D drawings and parameter information are consistent. For 3D-IDS files which are not for the first time to be countersigned, the system designers do not need to open the model and drawings to find the changes of the file, the changes will be shown in different colours. In both cases, the overall efficiency of the system designer will be greatly improved.

IDS file mechanical interface has only 3D model and parameter information extracted from the model. The consistency of them is guaranteed by the software algorithm, which does not require manual comparison, thus greatly liberating countersignature personnel and improving efficiency. The time and energy of countersign personnel are focused on whether the appearance and weight of equipment meet the requirements of system layout. This mode corrects the previous mode in which a great deal of effort was spent to ensure the consistency which should be ensured by software.

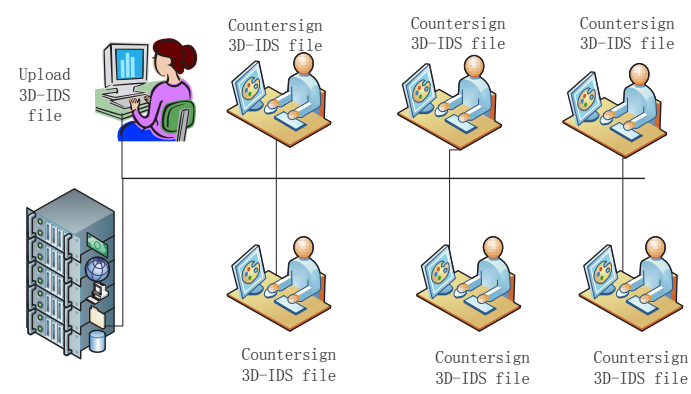

Fig. 5. Countersigning mode of IDS 


\subsection{D IDS file applications}

The model interface tool has the function of setting and verification. The model information and model inspection have been completed in the stage of model information extraction to ensure that all kinds of interface information have been set and all kinds of settings meet the needs of later stage.

In the mode of 3D-IDS, the system layout will be unified as follows: spacecraft designer downloads 3D model from controlled IDS files and uses the model for the spacecraft layout directly. No longer reset $\mathrm{R}$ coordinate system, installation interface, connector coordinate systems, thermistor coordinate systems, grounding coordinate systems, mass properties and other information.

Compared with the current pattern, there is no necessary to re-process the model, such as simplifying model, setting layout coordinate system, setting installation surface, setting lug thickness, setting mounting holes. Spacecraft designers will be liberated from repetitive, transactional work, so as to concentrate on layout design, assembly design, and improve the effectiveness of the work.

IDS file is one of basic references for equipment acceptance, and 3D-IDS files will be the main reference for equipment acceptance in the new mode. 3D-IDS files contain 3D models, which can clearly display the interface requirements for equipment acceptance and can be used as a reference for the physical acceptance of equipment. During the equipment acceptance, checkers inspect the physical equipment according to the holes position information, contour information and aperture information displayed in the 3D-IDS model.

\section{Conclusions}

This paper studies the application mode of 3D-IDS in spacecraft development. Based on the 3D-IDS system, the relevant information of the equipment interface can be automatically extracted from the 3D model and submitted to 3D-IDS file, the 3D model can be viewed visually, and the equipment shape, dimension and other related information can be viewed in real time. In this mode, the filling in and countersigning efficiency of IDS file are significantly improved, and the consistency is more easily guaranteed.

\section{References}

1. F.X. XU, Conspectus of Satellite Engineering. Beijing: China Astronautic Publishing House, 2003

2. C.R. PENG, System Design for Spacecraft. Beijing: China Science and Technology Press, 2011

3. Y.G. CHEN, An Introduction of Digital Design for Spacecraft. Beijing: China Science and Technology Press, 2010

4. P. WEI, L. WANG, H. ZHAO, Y. YUAN. Research on IDS Plug-in Mechanism for Spacecraft Multidisciplinary Data Acquisition and Processing. Aerospace Manufacturing Technology, 2016(2):5456.

5. W. YU, L. WANG, Z.J. WANG, P. WEI. Research on Interface Data Reuse of Spacecraft General Equipment. Aerospace Manufacturing Technology, 2017(2):62-64. 